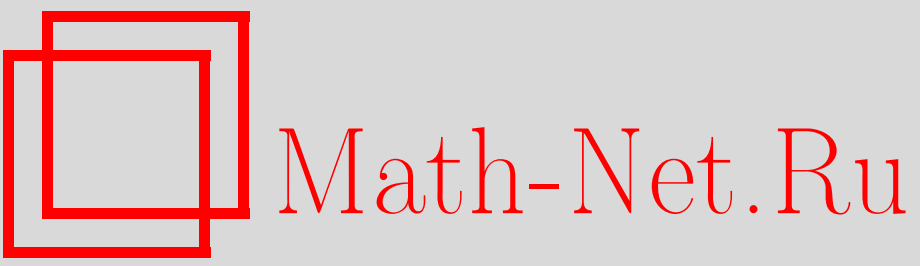

А. И. Никонов, Об одном свойстве взвешенных сумм одинаковых степеней как матричных произведений, Вестн. Сам. гос. техн. ун-та. Сер. Физ.-мат. наукu, 2010, выпуск 5(), 313-317

DOI: https://doi.org/10.14498/vsgtu816

Использование Общероссийского математического портала Math-Net.Ru подразумевает, что вы прочитали и согласны с пользовательским соглашением

http://www.mathnet.ru/rus/agreement

Параметры загрузки:

IP : 52.23 .180 .231

26 апреля 2023 г., $16: 13: 50$ 
УДК 512.14

\section{ОБ ОДНОМ СВОЙСТВЕ ВЗВЕШЕННЫХ СУММ ОДИНАКОВЫХ СТЕПЕНЕЙ КАК МАТРИЧНЫХ ПРОИЗВЕДЕНИЙ}

\section{А. И. Никонов}

Самарский государственный технический университет, 443100, Самара, ул. Молодогвардейская, 244.

E-mail: nikonovai@mail.ru

Конечная сумма взвешенных одинаковых степеней представлена в матричной борме. Это выражается наличием двух матричных компонентов, первый из которых не содержит, а второй содержит заданные весовые коэббициенты. Показательно, что если максимум степенных оснований в суммах рассматриваемого типа имеет значение не менъшее, чем значение степенного показателя, относящегося $к$ ее слагаемым, то первый матричный компонент является одинаковым для всех сумм, отвечающих указанному условию. Выявленное свойство общности матричного представления исполъзовано для получения модифицированной конечной суммы с увеличенным числом весовых коэфбициентов.

Ключевые слова: конечная сумма взвешенных одинаковых степеней, весовые коэфбициенты, матричный компонент, модифицированная конечная сумма.

Целью настоящей работы является представление свойства конечной взвешенной суммы одинаковых степеней, то есть степеней с одинаковыми показателями, которое позволяет повысить число степенных оснований приведения данной суммы к виду, использующему биномиальные коэффициенты [1] при сохранении её исходного значения.

Данное свойство, которое применимо, в частности, при передаче шифрованной информации в связи с возникновением потребности её объёмного расширения, может быть сформулировано на базе аппарата матричной алгебры. Тогда логично принять следующий план изложения нижеприведенного материала: описание представления известного преобразования взвешенной конечной суммы одинаковых степеней в матричной форме; рассмотрение сущности выявленного свойства общности конечной взвешенной суммы одинаковых степеней (при определённом ограничении); использование выявленного свойства для получения конечной взвешенной суммы одинаковых степеней с повышенным числом степенных оснований.

Исходную позицию нашего рассмотрения занимает конечная сумма

$$
\Phi(p, \nu)=\sum_{\pi=1}^{p} b_{\pi} \pi^{\nu} ; \quad p, \nu, \pi \in \mathbb{N} ; b_{\pi} \in \mathbb{R} .
$$

Поскольку речь здесь идет именно о сумме, считаем, что $p \geqslant 2, \nu \geqslant 1$.

Как известно [1], результат преобразования взвешенной суммы (1) содержит члены первого типа, не зависящие от коэффициентов вида $b_{\pi}$, а также члены второго типа, включающие в себя такие коэффициенты. Каждый из названных типовых членов определяется рекуррентно.

Произвольный член первого типа может быть представлен как $\alpha_{j(i+1)}^{i+1}$;

$$
\begin{gathered}
i \in\{0,1, \ldots, \max i\} \subset \mathbb{N}_{0}=\mathbb{N} \cup\{0\} ; \\
j(i+1) \in\{0,1, \ldots, \max j(i+1)\} \subset \mathbb{N}_{0} ;
\end{gathered}
$$

Александр Иванович Никонов (д.т.н., проф.), профессор, каф. электронных систем и информационной безопасности. 


$$
\max i=\min (p-1, \nu-1), \quad \max j(i+1)=\nu-(i+1)
$$

При этом

$$
\begin{gathered}
\alpha_{j(i+1)}^{i+1}=\sum_{j(i)=j(i+1)+1}^{\max j(i)} \alpha_{j(i)}^{i} C_{j(i)}^{j(i+1)}, \\
j(i) \in\{1,2, \ldots, \max j(i)\}, \quad \max j(i)=\nu-i ; \\
\alpha_{j(0)}^{0}= \begin{cases}0: & j(0)<\nu, \\
1: & j(0)=\nu .\end{cases}
\end{gathered}
$$

Для вхождения в итоговое выражение, которое отображает результат преобразования исходной суммы (1), отбираются члены первого типа, имеющие вид

$$
\alpha_{0}^{i+1}, \quad i \in\{0,1, \ldots, \max i\}
$$

Произвольный член второго типа, также входящий в указанное итоговое выражение, может быть представлен следующим образом:

$$
\begin{gathered}
\Phi_{p-(i+1) 0}=\sum_{\tau=0}^{p-(i+1)} b_{p-(i+1)-\tau}^{i+1}, \\
\tau \in\{0,1, \ldots, \max \tau\}, \quad \max \tau=p-(i+1) ; \\
b_{p-(i+1)-\tau}^{i+1}=b_{p-i-\tau}^{i}+b_{p-i-\tau}^{i+1} ; \\
\left.b_{p-i-\tau}^{i}\right|_{i=0}=b_{p-\tau}^{0}=b_{p-\tau} ; \\
b_{p-(i+1)-\tau}^{i+1}=\sum_{k=0}^{\tau} b_{p-i-k}^{i}, \quad i \geqslant 0 .
\end{gathered}
$$

В рамках настоящей работы всегда используются величины вида (4) со вторым нижним индексом, равным нулю. Данный вид далее будем обозначать просто через $\Phi_{p-(i+1)}$.

Итог преобразования суммы (1) задается выражением

$$
\Phi(p, \nu)=\sum_{i=0}^{\max i} \alpha_{0}^{i+1} \Phi_{p-(i+1)} .
$$

Вышеприведенные сведения о результате преобразования суммы (1), сопровождаемые соотношениями (3)-(4), с привлечением аппарата матричной алгебры [2] могут быть интерпретированы путем введения матричных компонентов данного результата: столбца и строки соответственно

$$
\begin{gathered}
M_{\alpha}=\left\|\alpha_{i+11}\right\|_{\max (i+1) \times 1} \\
M_{\phi}=\left\|\phi_{1 i+1}\right\|_{1 \times \max (i+1)} \\
\alpha_{i+11}=\alpha_{0}^{i+1}, \quad \phi_{1 i+1}=\Phi_{p-(i+1)} .
\end{gathered}
$$

Легко убедиться в том, что правая часть равенства (5) есть произведение матриц (6), (7). Матричный компонент (7), определяемый именно с участием исходных весовых коэффициентов вида $b_{\pi}$, будем обозначать через $M_{\Phi и}$.

Продолжая использование указанного аналитического аппарата, получаем следующее матричное представление соотношения (2):

$$
\alpha_{0}^{i+1}=\left\|c_{1 j(i)}\right\|_{1 \times \max j(i)}\left\|\alpha_{j(i) 1}\right\|_{\max j(i) \times 1} ;
$$




$$
\begin{gathered}
c_{1 j(i)}=C_{j(i)}^{0}, \alpha_{j(i) 1}=\alpha_{j(i)}^{i} ; \quad j(i)=1,2, \ldots, \max j(i) ; \\
\left\|\alpha_{j(i+1) 1}\right\|_{\max j(i+1) \times 1}=\left\|c_{j(i+1) j(i)}\right\|_{\max j(i+1) \times \max j(i)} \times \\
\times\left\|\alpha_{j(i) 1}\right\|_{\max j(i) \times 1} ; \quad c_{j(i+1) j(i)}=\sim \delta_{j(i) j(i+1)} C_{j(i)}^{j(i+1)}, \quad \alpha_{j(i+1) 1}=\alpha_{j(i+1)}^{i+1} .
\end{gathered}
$$

Здесь знак инверсии $\sim$ относится к $\delta_{j(i) j(i+1)}$.

В процессе определения левых частей (8), (9) для случая $p \geqslant \nu$ при $i=\max i$ строчный размер матрицы из левой части равенства (9) достигает нулевого значения. Использование данной матрицы и сам указанный процесс на этом прекращаются. Для случая $p \leqslant \nu$ указанный процесс завершается с приобретением матрицы из левой части $(9)$ размера $(\nu-p) \times 1$.

Рассмотрение совокупности соотношений (6), (8) позволяет выявить следующее свойство сумм взвешенных степеней: если $p \geqslant \nu$, то матричный компонент $M_{\alpha}$ либо соответствующий вектор, составленный из его элементов, является одинаковым для всех сумм, параметры $p$ и $\nu$ которых подчиняются приведенному неравенству, поскольку в данном случае величины вида $\alpha_{0}^{i+1}$ не зависят от значения $p$. Наличие такого свойства подтверждается самой сущностью формирования величин согласно выражениям (8), (9).

Например, при необходимости определения значений двух или более конечных сумм одинаковых степеней с различными количествами слагаемых (в частности, с параметрами $p_{1}$ и $p_{2} \neq p_{1}$ соответственно для двух конечных сумм) вычисление элементов матричных компонентов вида (6) следует произвести лишь однократно.

Далее поставим задачу, решение которой позволит проявить достоинство выявленного свойства матричного компонента $M_{\alpha}$ при $p \geqslant \nu$. Пусть требуется дополнить передаваемую информационную посылку - исходную совокупность весовых коэффициентов (весовую совокупность), используемую соответственно исходной конечной суммой одинаковых степеней заданного неизменного значения $\Phi(p, \nu)=\Phi_{3}$, несколькими вновь заданными весовыми коэффициентами. Кроме таких дополнительных коэффициентов, с целью поддержания неизменности $\Phi_{3}$ вместе с ними к исходной весовой совокупности добавляется также коэффициент $b_{b}$ вспомогательного характера, значение которого необходимо выяснить.

Поставленная задача должна быть решена без какой-либо вставки вспомогательного коэффициента $b_{b}$ внутрь исходной или вновь формируемой весовой совокупности и, соответственно, без вставки слагаемого со вспомогательным коэффициентом внутрь массива слагаемых одинаковых степеней с исходными и дополнительными весовыми коэффициентами. Условимся получить решение данной задачи, сделав величину $b_{b}$ весовым коэффициентом при слагаемом из модифицированной конечной суммы $\Phi(\rho, \nu)=\Phi_{3}(\rho \in \mathbb{N}, \rho>p)$ с наименьшим - единичным основанием.

Отметим, что $i$ является, по сути дела, индексом порядкового снижения [1]: каждое из значений $\max j(i), \max j(i+1)$ после проведения действий $(8),(9)$ снижается на единицу. Величину $\Phi_{p-i}$ будем именовать пооперационной суммой, причём именно она зависит от весовых коэффициентов.

Модифицированная пооперационная сумма $\Phi_{\rho-i}$ зависит соответственно от модифицированных весовых коэффициентов вида $b_{M \pi}$ :

$$
b_{M \pi}= \begin{cases}b_{M 1}=b_{b}: & \pi=1 \\ b_{M \pi}=b_{\pi-1}: & \pi=2,3, \ldots, p+1 \\ b_{M \pi}=b_{g \pi-p-1}: & \pi=p+2, p+3, \ldots, \rho,\end{cases}
$$

где $b_{g \pi-p-1} \in\left\{b_{g 1}, b_{g 2}, \ldots, b_{g \rho-p-1}\right\}$ - дополнительно вводимый заданный весовой коэффициент.

Такая зависимость для произвольного индекса порядкового снижения может быть представлена как

$$
\Phi_{\rho-(i+1)}=\Phi_{\rho-(i+1)}\left(b_{M 0}^{i+1}, b_{M 1}^{i+1}, \ldots, b_{M \rho-(i+1)}^{i+1}\right),
$$


а поскольку по рекуррентной цепочке любое $b_{M \rho-(i+1)-\tau}^{i+1}$ выражается через $b_{M 1}, \ldots$, $b_{M \rho}$, справедливо также равенство

$$
\Phi_{\rho-(i+1)}=\Phi_{\rho-(i+1)}\left(b_{M 1}, \ldots, b_{M \rho}\right) .
$$

Аддитивность построения величины $\Phi_{\rho-(i+1)}$ применительно к рассматриваемому случаю можно выразить следующим образом:

$$
\Phi_{\rho-(i+1)}=\Phi_{\rho-(i+1)}\left(b_{M 1}=0\right)+\Phi_{\rho-(i+1)}\left(b_{b}, 0, \ldots, 0\right),
$$

причём $\Phi_{\rho-(i+1)}\left(b_{M 1}=0\right)=\Phi_{\rho-(i+1)}\left(0, b_{M 2}, \ldots, b_{M p+1}, b_{g 1}, \ldots, b_{g \rho-(p+1)}\right)$.

Последнее слагаемое из (10) составляет 0 для любого $(i+1)>1$ и $b_{b}$ для $(i+1)=1$.

Тогда требуемый баланс исходно заданного значения $\Phi_{3}=\Phi(p, \nu)=M_{\Phi и} M_{\alpha}$ и модифицированной конечной суммы $\Phi(\rho, \nu)$ имеет вид

$$
\Phi_{3}=M_{\Phi И} M_{\alpha}=M_{\Phi M} M_{\alpha}+b_{b}
$$

где $M_{\Phi M}=\left\|\phi_{1 i+1}^{M}\right\|_{1 \times \max (i+1)} ; \phi_{1 i+1}^{M}=\Phi_{\rho-(i+1)}\left(b_{M 1}=0\right)$.

Следовательно, искомое значение вспомогательного коэффициента составляет

$$
b_{b}=\Phi_{3}-M_{\Phi M} M_{\alpha}
$$

или

$$
b_{b}=\left(M_{\Phi И}-M_{\Phi M}\right) M_{\alpha}=\left\|\phi_{1 i+1}-\phi_{1 i+1}^{M}\right\|_{1 \times \max (i+1)} M_{\alpha} .
$$

Как видим, при определении искомого вспомогательного коэффициента матричный компонент $M_{\alpha}$ использован однократно.

Сделаем еще одно замечание, связанное с выявленным свойством преобразования суммы взвешенных одинаковых степеней. В каком-либо конкретном случае применения результатов такого преобразования его пользователь может прийти к заключению о недостаточной уникальности значения матричного компонента $M_{\alpha}$.

С целью придания данной матрице больших отличительных свойств, в частности, известных только упомянутому пользователю, им может быть принят к рассмотрению следующий вид исходной конечной суммы одинаковых степеней:

$$
\Phi(p \chi, \nu)=\sum_{\pi=1}^{p} b_{\pi}(\pi \chi)^{\nu} ; \quad p, \nu, \pi \in \mathbb{N} ; b_{\pi}, \chi \in \mathbb{R} .
$$

Связь данного вида с исходной конечной суммой (1) такова:

$$
\Phi(p \chi, \nu)=\chi^{\nu} \Phi(p, \nu) .
$$

Тогда произвольно выделяемые пользователем в величине $\chi^{\nu}$ сомножители $\chi_{1}$ и $\chi_{2}\left(\chi^{\nu}=\chi_{1} \chi_{2} ; \chi_{1}, \chi_{2} \in \mathbb{R}\right)$ при определении значения $\Phi(p \chi, \nu)$ могут мультипликативно добавляться к матричным компонентам суммы $\Phi(p, \nu)$, придавая им тем самым большую числовую обособленность. 


\section{БИБЛИОГРАФИЧЕСКИЙ СПИСОК}

1. Никонов А.И. Преобразование суммы взвешенных степеней натуральных чисел с одинаковыми показателями // Вестн. Сам. гос. техн. ун-та. Сер. Физ.-мат. науки, 2010.№ 1(20). - С. 258-262.

2. Стренг Г. Линейная алгебра и её применения. - М.: Мир, 1980. - 456 с.

Поступила в редакцию 07/VII/2010; в окончательном варианте - 30/IX/2010.

MSC: 11C20, 05A10

ON ONE PROPERTY OF THE WEIGHED SUMS OF EQUAL POWERS AS MATRIX PRODUCTS

\section{A.I. Nikonov}

Samara State Technical University,

244, Molodogvardeyskaya st., Samara, 443100, Russia.

E-mail: nikonovai@mail.ru

Finite sum of weighted sum of equal powers is represented in matrix form. This is expressed by the presence of two matrix components, the first of which does not contain, and the second contains the specified weights. It is significant that if the maximum power grounds in the amounts of this type has a value of no less important exponent related to its term, the first matrix component is the same for all amounts that meet the specified condition. Revealed common property of the matrix representation used to obtain the modified final sum from the increased number of weights.

Key words: finite sum of the weighed equal powers, weight factors, matrix component, modified finite sum.

Original article submitted 07/VII/2010; revision submitted 30/IX/2010.

Alexander I. Nikonov (Dr. Sci. (Techn.)), Professor, Dept. of Electronic Systems \& Information Security. 